\title{
Downregulated ZC3H13 by miR-362-3p/miR-425-5p is Associated with Poor Prognosis and Adverse Outcomes in Hepatocellular Carcinoma
}

\section{shuang wu}

The Affiliated Hospital of Qingdao University https://orcid.org/0000-0003-1988-1410

Shihai Liu

The Affiliated Hospital of Qingdao University

\section{Yongxian Cao}

The Affiliated Hospital of Qingdao University

Chao Geng

The Affiliated Hospital of Qingdao University

\section{Peng Wang}

Weifang Yidu Central Hospital

Huazheng Pan ( $\sim$ panhuazheng@126.com )

The Affiliated Hospital of Qingdao University https://orcid.org/0000-0001-6604-2770

\section{Primary research}

Keywords: ZC3H13, N6-Methyladenosine, Hepatocellular carcinoma, Tumor immune infiltration, Prognosis

Posted Date: November 2nd, 2021

DOI: https://doi.org/10.21203/rs.3.rs-1015202/v1

License: (c) (i) This work is licensed under a Creative Commons Attribution 4.0 International License. Read Full License 


\section{Abstract}

Background: Hepatocellular carcinoma (HCC) is notorious for its poor prognosis. Increasing evidence has demonstrated that N6-methyladenosine $(\mathrm{m} 6 \mathrm{~A})$ related genes plays key roles in initiation and progression of several types of human cancer. However, the specific role and mechanism of m6A RNA modification in HCC remains not fully determined. Previous studies identified that several m6A-related genes, especially $\mathrm{ZC} 3 \mathrm{H} 13$, could be a high candidate as a novel biomarker and therapeutic target for hepatocellular carcinoma. In liver hepatocellular carcinoma (LIHC), the low expression of $\mathrm{ZC} 3 \mathrm{H} 13$ was reported but the molecular reason is unclear.

Methods: Oncomine and GEPIA databases were used to explore the differential expression of ZC3H13 in multiple cancers. Kaplan-Meier plotter was selected to explore the prognostic value of ZC3H13. The GSCALite database was used to analyze the genetic variation of the m6A-related genes. StarBase was selected to predict and analysis of upstream miRNAs of ZC3H13. MiR-362-3p/miR-425-5p mimics and inhibitors results detected by Quantitative Real-time PCR (qPCR) analysis and western blotting. Gene set enrichment analysis (GSEA) identified the potential regulatory mechanism of ZC3H13. In addition, we investigated the relationship between $\mathrm{ZC} 3 \mathrm{H} 13$ and tumor infiltrating immune cells (TIICs). Finally, we determined the expression correlation of $\mathrm{ZC} 3 \mathrm{H} 13$ with biomarkers of immune cells in HCC using GEPIA database.

Results: In this study, we found that ZC3H13 expression was significantly correlated with both overall survival (OS) and progression-free survival (RFS) in LIHC patients based on a comprehensive analysis of the m6A family. Our experimental results indicate that inhibiting miR-362-3p/miR-425-5p expression in the $\mathrm{LIHC}$ cell line significantly restored the expression of $\mathrm{ZC} 3 \mathrm{H} 13$, which is consistent with bioinformatic studies. Further, we noticed that there is a possible relationship between $\mathrm{ZC} 3 \mathrm{H} 13$ high expression and tumor microenvironment infiltrating immune cells.

Conclusions: In conclusion, this study demonstrates that $\mathrm{ZC} 3 \mathrm{H} 13$ is a direct target of miR-362-3p/miR425-5p in LIHC that regulates the immune modulations in the microenvironment of LIHC.

\section{Introduction}

Hepatocellular carcinoma (HCC) is the most common type of primary liver cancer and also ranks as the third leading cause of cancer-related deaths all over the world [1]. Established risk factors for liver cancer are limited to chronic hepatitis B and C virus (HBV/HCV) infections, obesity, type 2 diabetes, alcohol abuse, smoking and aflatoxin [2]. The onset of liver cancer is occult and most of the symptoms will not appear until the middle and late stages, thus making it rather difficult to diagnose liver cancer at the early stage [3]. Despite the huge improvements that have been achieved in aspects of diagnosis, therapy [4], and prognosis, the outcome of patients with HCC remains unsatisfactory, with more than 700,000 deaths every year [5]. Therefore, it is an urgent need to develop effective therapeutic targets or seek promising prognostic biomarkers in HCC. 
The occurrence and development of tumors are driven by the disorder of genetic, epigenetic, and environmental factors, and epigenetic factors play an important role as a bridge between genetic and environmental factor [6]. RNA modifications are also important types of post-transcriptional epigenetic modification, and N6-methyladenosine (m6A) is the most predominant modification of mRNA $[7,8]$. In mammalian cells, there is an average of $1-2 \mathrm{~m} 6 \mathrm{~A}$ sites per 1,000 nucleotides. At present, many proteins involved in m6A have been identified, which are classified into m6A methylation transferases ("writer" proteins), m6A demethylases ("eraser" proteins), and m6A reading and binding proteins ("reader" proteins) [9]. RNA is methylated at the sixth nitrogen atom of adenylate by the action of "writer" proteins, and methyltransferase-like 3 (METTL3) and methyltransferase-like 14 (METTL14) form a hetero complex, which complete this modification process together with Wilms tumor 1 associated protein (WTAP) and other factors, such as Putative RNA-binding protein 15 (RBM15) and Zinc Finger CCCH-Type Containing 13 (ZC3H13) [10]. By contrast, the m6A modified regions can be demethylated under the action of two "eraser" proteins, fat mass, and obesity-associated protein (FTO) and alkB homolog 5 (ALKBH5) making $\mathrm{m} 6 \mathrm{~A}$ a reversible modification. These m6A modified sites can be recognized by "readers" proteins to regulate RNA metabolism, including translation, splicing, translocation, degradation, and processing. $\mathrm{ZC} 3 \mathrm{H} 13$ included in "writers" have also been reported to be involved in cancers. For example, Gong et al demonstrated that downregulation of METTL14 and ZC3H13 which act as two tumor suppressor genes was found in breast cancer and predicted poor prognosis. Their abnormal expression promoted breast cancer invasion by affecting pathways related to tumor progression and mediating immunosuppression [11]. A recent report published by the team of Zhu et al has also validated that ZC3H13 may be an upstream regulator of Ras-ERK signaling pathway and suppressed invasion and proliferation of colorectal cancer [12].However, a comprehensive study regarding the expression, prognosis, and mechanism of $\mathrm{ZC} 3 \mathrm{H} 13$ in $\mathrm{HCC}$ is still absent. Moreover, the association of $\mathrm{ZC} 3 \mathrm{H} 13$ with tumor immune infiltration in $\mathrm{HCC}$ is still not determined.

The post-transcriptional alteration of mRNAs is one of the most well-known mechanisms by which miRNAs regulate their target genes. Although the $\mathrm{ZC} 3 \mathrm{H} 13$ deficiency is widely documented, there has been no research on $\mathrm{ZC} 3 \mathrm{H} 13$ dependent mRNA expression and post-transcriptional repression by noncoding miRNAs in LIHC. To address this gap, we searched for miRNAs-targeted ZC3H13 in TCGA-based data tools and found a subset of miRNAs that may target ZC3H13 in liver cancer. We also uncovered the prognosis significance of low $\mathrm{ZC} 3 \mathrm{H} 13$ levels in liver cancer, using RNA sequencing data from the TCGA database. We discovered that $\mathrm{ZC} 3 \mathrm{H} 13$ predicted poor prognosis in liver cancer patients.

\section{Materials And Methods}

\section{Candidate miRNA prediction}

Upstream binding miRNAs of $\mathrm{ZC} 3 \mathrm{H} 13$ were predicted by several target gene prediction programs, consisting of PITA, RNA22, miRmap, microT, miRanda, PicTar, and TargetScan. Only the predicted miRNAs that commonly appeared in more than two programs as mentioned above were included for subsequent analyses. These predicted miRNAs were regarded as candidate miRNAs of ZC3H13. 


\section{Oncomine analysis}

The Oncomine database (http://www.oncomine.org) is an online microarray database that includes 715 datasets, as well as 86,733 cancer and normal tissue samples [13]. In this study, the Oncomine database was employed to analyze the mRNA expression levels of m6A-related genes in hepatocellular carcinoma and liver tissue. The search was carried out based on the following criteria: (a) type of analysis: cancer versus normal tissues; (b) type of data: mRNA; (c) thresholds: fold change $=2$ and $\mathrm{P}$ value $=0.01$.

\section{GEPIA database analysis}

GEPIA (http://gepia.cancer-pku.cn/) is a web tool for cancer and normal gene-expression profiling and interactive analyses based on TCGA and The Genotype-Tissue Expression (GTEx) data [14]. GEPIA was used to determine $\mathrm{ZC} 3 \mathrm{H} 13$ expression in various types of human cancer. $\mathrm{p}$ value $<0.05$ was considered as statistically significant. In addition, expression correlation of $\mathrm{ZC} 3 \mathrm{H} 13$ with immune checkpoints in HCC was also evaluated using GEPIA database. $|\mathrm{R}|>0.1$ and $p$ value $<0.05$ were set as selection criteria for identifying as statistically significant.

\section{Kaplan-Meier plotter analysis}

As a tool for assessing biomarkers, the Kaplan-Meier plotter (http://kmplot.com) can be used to assess the functions of 54,675 genes and 10,188 tumor tissue samples, including breast cancer, liver cancer, lung cancer and gastric cancer [15]. It was employed to conduct survival analysis for ZC3H13 in BRCA, KIRC, LIHC, LUAD and STAD as we previously described. Log rank $p$ value $<0.05$ was considered as statistically significant.

\section{StarBase databaqse analysis}

StarBase (http://starbase.sysu.edu.cn/) is a database for exploring miRNA-related studies [16]. StarBase was introduced to perform expression correlation analysis for miR-362-3p/miR-425-5p-ZC3H13 in HCC. The expression level of miR-362-3p/miR-425-5p in HCC and normal controls was also analyzed by StarBase.

\section{TIMER database analysis}

TIMER (https://cistrome.shinyapps.io/timer/) is a web server for comprehensive analysis of tumorinfiltrating immune cells [17]. TIMER was used to analyze the correlation of ZC3H13 expression level with immune cell infiltration level or immune checkpoint expression level in HCC. $p$ value $<0.05$ was considered as statistically significant. 


\section{LinkedOmics}

LinkedOmics (http://www.linkedomics.org) is a publicly available portal that includes multi-omics data from all 32 TCGA Cancer types [18]. In the "LinkFinder" module of LinkedOmics, we used the Pearson test to perform statistical analysis on $\mathrm{ZC} 3 \mathrm{H} 13$ co-expression, and the results were displayed in the form of volcano, heat, or scatter plots. "LinkInterpreter" module of LinkedOmics was applied to conducted analyses of Gene Ontology (Biological Process), Kyoto Encyclopedia of Genes and Genomes (KEGG) pathways through Gene Set Enrichment Analysis (GSEA). The rank criterion was false discovery rate $(\mathrm{FDR})<0.05$, and simulations was 500 .

\section{UALCAN}

UALCAN is designed for gene expression analysis, prognosis analysis, and methylation analysis and is based on data of The Cancer Genome Atlas Program (TCGA) [19]. TCGA is a landmark cancer genomics program, and molecularly characterized over 20,000 primary cancer and matched normal samples spanning 33 cancer types. Gene expression of miR-362-3p/miR-425-5p in LIHC TCGA dataset were explored using UALCAN. $\mathrm{P}<0.05$ indicated statistical significance.

\section{Cell Culture, Cell Transfection, RNA Isolation and Quantitative Real-time PCR (qPCR) analysis}

The HCC cell lines used in this study were purchased from American Type Culture Collection. These cell lines include Hep3B and Huh7 cells. All cancer cells were maintained in high-glucose Dulbecco's modified Eagle medium (DMEM; Thermo Scientific) supplemented with $10 \%$ fetal bovine serum (FBS, Gibco), 0.1 $\mathrm{mmol} / \mathrm{L}$ MEM nonessential amino acids (NEAA; Invitrogen), and 1\% L-glutamine (Invitrogen). All of the cell lines were cultured in $5 \% \mathrm{CO}_{2}$ at $37^{\circ} \mathrm{C}$ in incubators at $100 \%$ humidity. Cells at the density of $2 \times 10^{6} / \mathrm{ml}$ were transfected with indicated miRNA mimics or inhibitors using Lipofectamine ${ }^{\circledR} 3000$ (Invitrogen; Thermo Fisher Scientific, Inc.) according to the manufacturer's instructions. At $48 \mathrm{~h}$ posttransfection, cells were used for subsequent experiments.

Total RNA was extracted from HCC cells using TRIzol® reagent (Invitrogen; Thermo Fisher Scientific, Inc.). Total RNA $(1 \mu \mathrm{g})$ was reverse transcribed into cDNA $\left(37^{\circ} \mathrm{C}\right.$ for $15 \mathrm{~min}$ and $85^{\circ} \mathrm{C}$ for $\left.5 \mathrm{sec}\right)$ using the PrimeScript RT reagent kit with gDNA Eraser (Takara Biotechnology Co., Ltd.). Subsequently, qPCR was performed using the TB Green Fast qPCR mix (Takara Biotechnology Co., Ltd.). The following primers were used for qPCR: ZC3H13 forward, 5'- AAAGGAGGTTTCACCAGAAGTG-3' and reverse, 5'CGCTTCGGAGATTTGCTAGAC -3'; and GAPDH forward, 5'- GAAATCCCATCACCATCTTCCAGG -3' and reverse, 5'- GAGCCCCAGCCTTCTCCATG -3'. The following thermocycling conditions were used for qPCR: Initial denaturation at $95^{\circ} \mathrm{C}$ for $2 \mathrm{~min}$; followed by 40 cycles at $95^{\circ} \mathrm{C}$ for $20 \mathrm{sec}, 58^{\circ} \mathrm{C}$ for $20 \mathrm{sec}$ and $72^{\circ} \mathrm{C}$ 
for $20 \mathrm{sec}$. mRNA expression levels were quantified using the $2-\Delta \Delta \mathrm{Ct}$ method and normalized to the internal reference gene GAPDH.

\section{Western Blotting}

Cells were collected and washed with PBS twice. Cells were then lysed with RIPA lysis buffer containing protein inhibitors on ice for 30 minutes. Cell lysis was then spun down at $13000 \mathrm{rpm}$ for 30 minutes. Supernatant containing protein was collected, and protein was quantitated with Pierce BCA Protein Assay Kit (Thermo Fisher Scientific, Waltham, MA). SDS-PAGE was used to separate proteins by electrophoresis based on protein size. After electrophoresis, protein was transferred to Polyvinylidene difluoride (PVDF) membrane. Membrane was blotted with $5 \%$ non-fat milk at room temperature with shaking for 30 minutes. Primary antibodies targeting proteins of interest were incubated with membrane with shaking at $4^{\circ} \mathrm{C}$ overnight. The next day, HRP-tagged secondary antibodies were used to detect proteins of interest. Finally, the bands were illuminated with Pierce ECL (Thermo Fisher Scientific, Waltham, MA). All of the images were captured using the Molecular Imager ChemiDoc XRS+ (Bio-Rad, Hercules, CA).

\section{Statistical analysis}

The statistical analysis in this study was automatically calculated by the online database mentioned above. $p$ value $<0.05$ or log rank $p$ value $<0.05$ was considered as statistically significant.

\section{Results}

\section{Pan-cancer analysis of ZC3H13 expression}

To explore possible roles of $\mathrm{ZC} 3 \mathrm{H} 13$ in carcinogenesis, we first analyzed its expression in 20 types of human cancer using Oncomine database. As shown in Figure 1A, compared with normal samples, ZC3H13 was significantly upregulated in 5 cancer types, including colorectal cancer, kidney cancer, lymphoma, melanoma, and sarcoma, and was markedly downregulated in 4 cancer types, involving brain and CNS cancer, liver cancer, lung cancer, and lymphoma. Next, we further validated the expression of ZC3H13 in these 33 cancer types using GEPIA database. As presented in Figure 1B, ZC3H13 expression in DLBC, ESCA, LAML, PAAD, SKCM, STAD, and THYM was statistically increased when compared with corresponding normal controls. And in LIHC, ZC3H13 was obviously decreased (Figure 1C). Taken together, $\mathrm{ZC} 3 \mathrm{H} 13$ was downregulated in $\mathrm{LIHC}$, indicating that $\mathrm{ZC} 3 \mathrm{H} 13$ may function as crucial regulator in carcinogenesis of the liver cancer.

\section{The prognostic values of $\mathrm{ZC} 3 \mathrm{H} 13$ in human cancer}

Next, survival analysis for ZC3H13 in BRCA, KIRC, LIHC, LUAD and STAD was conducted. Two prognostic indices, consisting of overall survival (OS) and progression-free survival (RFS), were included. For OS, 
$\mathrm{KIRC}$ and $\mathrm{LIHC}$ patients with higher expression of $\mathrm{ZC} 3 \mathrm{H} 13$ indicated better prognosis (Figures $2 \mathrm{C}$, E). For RFS, among all cancer types, only increased expression of $\mathrm{ZC} 3 \mathrm{H} 13$ indicated poor prognosis in $\mathrm{LIHC}$ (Figure 2F). No statistical significance of $\mathrm{ZC} 3 \mathrm{H} 13$ for predicting prognosis of patients in other cancer types was observed. By combination of OS and RFS, ZC3H13 may be utilized as an unfavorable prognostic biomarker in patients with HCC.

\section{Prediction and analysis of upstream miRNAs of ZC3H13}

It has been widely acknowledged that ncRNAs are responsible for the regulation of gene expression. To ascertain whether $\mathrm{ZC} 3 \mathrm{H} 13$ was modulated by some ncRNAs, we first predicted upstream miRNAs that could potentially bind to $\mathrm{ZC} 3 \mathrm{H} 13$ and finally found 12 miRNAs (Table 1). To improve visualization, a miRNA-ZC3H13 regulatory network was established using cytoscape software (Figure $3 \mathrm{~A}$ ). Based on the action mechanism of miRNA in regulation of target gene expression, there should be negative correlation between miRNA and ZC3H13.Thus, the expression correlation analysis was performed. As showed in Figures $3 \mathrm{~B}$ and $3 \mathrm{E} \mathrm{ZC} 3 \mathrm{H} 13$ was significantly negatively correlated with miR-362-3p or miR-425-5p in HCC. There were no statistical expression relationships between $\mathrm{ZC} 3 \mathrm{H} 13$ and the other 9 predicted miRNAs. Based on the ceRNA hypothesis, miRNA expression was also upregulated in liver cancer wherein it negatively correlates with $\mathrm{ZC} 3 \mathrm{H} 13$. These results are consistent with bioinformatics analysis. Expression analysis of miR-362-3p/ miR-425-5p based on the TCGA database revealed miR-362-3p/miR-425-5p was upregulated in $\mathrm{HCC}$ tissue. As Figures $3 \mathrm{C}$ and $3 \mathrm{~F}$ shows, there is a significant difference between the two groups. Finally, the expression and prognostic value of miR-362-3p/ miR-425-5p in HCC were determined. As presented in Figures $3 \mathrm{C}$ and $3 \mathrm{G}$, miR-362-3p/ miR-425-5p was markedly upregulated in HCC and its downregulation was positively linked to patients'prognosis. Huh7 and Hep3B cells were transfected with miR-362-3p/ miR-425-5p and checked for the impact on ZC3H13 levels. We found that miR-362-3p/ miR425-5p mimics significantly reduced the $\mathrm{ZC} 3 \mathrm{H} 13$ level compared with $\mathrm{NC}$, and the reduction was repealed by miR-362-3p/ miR-425-5p inhibitors as evidenced by qRT-PCR (Figures 3H, I). Additionally, we performed a western blotting analysis to examine the $\mathrm{ZC} 3 \mathrm{H} 13$ protein level. The results showed that miR-362-3p/ miR-425-5p mimics and inhibitors significantly decreased and increased ZC3H13 protein levels, respectively (Figure $3 \mathrm{~J}$ ). The most striking result to emerge from the data in Figure 3 is that $\mathrm{ZC} 3 \mathrm{H} 13$ is a direct target of miR-362-3p/ miR-425-5p and is tumor suppressor gene involved in HCC tumor progression, which further confirmed RNA-seq results. 


\begin{tabular}{|llll|}
\hline \multicolumn{4}{|c|}{ Table 1 } \\
\hline \multicolumn{4}{|c|}{ The expression correlation between predicted miRNAs and ZC3H13 in HCC. } \\
\hline geneName & miRNAname & R-value & P-value \\
\hline ZC3H13 & hsa-miR-15a-5p & 0.069 & 0.183 \\
\hline ZC3H13 & hsa-miR-16-5p & $0.173^{a}$ & $8.29 \mathrm{E}-04^{a}$ \\
\hline ZC3H13 & hsa-miR-23a-3p & 0.081 & 0.119 \\
\hline ZC3H13 & hsa-miR-7-5p & 0.026 & 0.616 \\
\hline ZC3H13 & hsa-miR-15b-5p & 0.021 & 0.691 \\
\hline ZC3H13 & hsa-miR-23b-3p & 0.036 & 0.492 \\
\hline ZC3H13 & hsa-miR-195-5p & $0.173^{a}$ & $8.35 \mathrm{E}-04^{\mathrm{a}}$ \\
\hline ZC3H13 & hsa-miR-424-5p & 0.086 & 0.1 \\
\hline ZC3H13 & hsa-miR-329-3p & 0.006 & 0.905 \\
\hline ZC3H13 & hsa-miR-497-5p & 0.079 & 0.130 \\
\hline ZC3H13 & hsa-miR-425-5p & $-0.129^{a}$ & $0.0128^{a^{*}}$ \\
\hline ZC3H13 & hsa-miR-362-3p & $-0.106^{a}$ & $0.0417^{a^{*}}$ \\
\hline a These results are statistially significant. & & \\
\hline *p value <0.05; **p value <0.01; ***p value < 0.001. & \\
\hline
\end{tabular}

\section{Determining the function of $\mathrm{ZC} 3 \mathrm{H} 13$ in liver cancer}

To understand the function of $\mathrm{ZC} 3 \mathrm{H} 13$ in $\mathrm{LIHC}$, we utilized available TCGA sequencing data using LinkedOimcs online tool. The association results and top 50 positively and negatively correlated genes are shown in Additional file 1. As plotted in Figure 4A, it shows that red dots positively correlated with $\mathrm{ZC} 3 \mathrm{H} 13$, and green dots negatively correlated ( $p$-value $<0.05$ ). KEGG analysis showed genes were primarily enriched in the JAK-STAT signaling pathway, hippo signaling pathway, inositol phosphate metabolism, chagas disease, propanoate metabolism and transcriptional misregulation in cancer.

\section{ZC3H13 positively correlates with immune cell infiltration in HCC}

$\mathrm{ZC} 3 \mathrm{H} 13$ is a member of m6A-related genes, which have an immunoglobulin domain and are reported to play a critical role in the immune system. As shown in Figure 5A, no significant change of immune cell 
infiltration level under various copy numbers of $\mathrm{ZC} 3 \mathrm{H} 13$ in $\mathrm{HCC}$ was observed. Correlation analysis could provide key clues for studying the function and mechanism of $\mathrm{ZC} 3 \mathrm{H} 13$. Thus, the correlation of $\mathrm{ZC} 3 \mathrm{H} 13$ expression level with immune cell infiltration level was evaluated. As presented in Figures 5B-5G, ZC3H13 expression was significantly positively associated with four kinds of immune cells, including CD4+T cell, macrophage, neutrophil, and dendritic cell in HCC, such a correlation was not seen for CD8+T cell and B cell.

\section{Expression correlation of ZC3H13 and biomarkers of immune cells in HCC}

To further explore the role of $\mathrm{ZC} 3 \mathrm{H} 13$ in tumor immune, we determined the expression correlation of ZC3H13 with biomarkers of immune cells in HCC using GEPIA database. As listed in Table 2, ZC3H13 was significantly positively correlated with CD4+T cell's biomarker (CD4), M1 macrophage's biomarkers (NOS2, IRF5, and PTGS2), M2 macrophage's biomarkers (VSIG4, and MS4A4A), neutrophil's biomarkers (ITGAM and CCR7), and dendritic cell's biomarkers (HLA-DPB1, HLA-DRA, HLA-DPA1, CD1C, NRP1, and ITGAX) in HCC, but B cell's biomarkers (CD79A), CD8+T cell's biomarkers need further validation. These findings partially support that $\mathrm{ZC} 3 \mathrm{H} 13$ is positively linked to immune cell infiltration. 


\begin{tabular}{|c|c|c|c|}
\hline \multicolumn{4}{|c|}{$\begin{array}{rr}\text { Table } 2\end{array}$} \\
\hline \multicolumn{4}{|c|}{ Correlation analysis between $\mathrm{ZC} 3 \mathrm{H} 13$ and biomarkers of immune cells in $\mathrm{HCC}$. } \\
\hline Immune cell & Biomarker & $R$ value & $\mathrm{p}$ value \\
\hline \multirow[t]{2}{*}{ B cell } & CD19 & -0.013 & 8.00E-01 \\
\hline & CD79A & $0.1^{\mathrm{a}}$ & $4.80 \mathrm{E}-02^{\star a}$ \\
\hline \multirow[t]{2}{*}{ CD8+ T cell } & CD8A & $0.15^{\mathrm{a}}$ & $4.20 \mathrm{E}-03^{\star *} \mathrm{a}$ \\
\hline & CD8B & 0.033 & $5.20 \mathrm{E}-01$ \\
\hline CD4+ $\mathrm{T}$ cell & CD4 & $0.25^{\mathrm{a}}$ & $1.60 \mathrm{E}-06^{\star \star \star} \mathrm{a}$ \\
\hline \multirow[t]{3}{*}{ M1 macrophage } & Nos2 & $0.29^{a}$ & $1.70 \mathrm{E}-08 * \star \star a$ \\
\hline & IRF5 & $0.2^{\mathrm{a}}$ & $7.50 \mathrm{E}-05^{\star \star \star} \mathrm{a}$ \\
\hline & PTGS2 & $0.4^{\mathrm{a}}$ & $1.20 \mathrm{E}-15^{\star \star \star} \mathrm{a}$ \\
\hline \multirow[t]{3}{*}{ M2 macrophage } & CD163 & 0.07 & $1.80 \mathrm{E}-01$ \\
\hline & VSIG4 & $0.18^{a}$ & $3.90 \mathrm{E}-04^{\star * \star} \mathrm{a}$ \\
\hline & MS4A4A & $0.25^{\mathrm{a}}$ & $1.10 \mathrm{E}-06^{\star \star \star \star} \mathrm{a}$ \\
\hline \multirow[t]{3}{*}{ Neutrophil } & CEACAM8 & 0.078 & $1.30 \mathrm{E}-01$ \\
\hline & ITGAM & $0.24^{\mathrm{a}}$ & $2.30 \mathrm{E}-06^{\star \star \star} \mathrm{a}$ \\
\hline & CCR7 & $0.25^{\mathrm{a}}$ & $7.50 \mathrm{E}-07 * \star \star a$ \\
\hline \multirow[t]{7}{*}{ Dendritic cell } & HLA-DPB1 & $0.17^{a}$ & $1.10 \mathrm{E}-03 * \star \mathrm{a}$ \\
\hline & HLA -DQB1 & -0.078 & $1.30 \mathrm{E}-01$ \\
\hline & HLA-DRA & $0.22^{\mathrm{a}}$ & $1.60 \mathrm{E}-05^{\star \star \star} \mathrm{a}$ \\
\hline & HLA-DPA1 & $0.26^{\mathrm{a}}$ & $4.00 \mathrm{E}-07 \star \star \star a$ \\
\hline & CD1C & $0.2^{\mathrm{a}}$ & $1.20 \mathrm{E}-04^{\star \star \star} \mathrm{a}$ \\
\hline & NRP1 & $0.5^{\mathrm{a}}$ & $1.40 \mathrm{E}-24 * \star \star a$ \\
\hline & ITGAX & $0.28^{a}$ & $7.60 \mathrm{E}-08^{\star \star \star} \mathrm{a}$ \\
\hline $\begin{array}{l}\text { aThese results ar } \\
{ }^{*} \mathrm{p} \text { value }<0.05 ; *\end{array}$ & $\begin{array}{l}\text { Illy significe } \\
<0.01 ; * \star \star p\end{array}$ & 0.001 & \\
\hline
\end{tabular}




\section{Relationship between ZC3H13 and immune checkpoints in HCC}

PD1/PD-L1 and CTLA-4 are important immune checkpoints that are responsible for tumor immune escape. Considering the potential tumor suppressor gene of ZC3H13 in HCC, the relationship of ZC3H13 with PD1, PD-L1, or CTLA-4 was assessed. As suggested in Additional file 2, ZC3H13 expression was significantly positively correlated with PD-L1, in HCC, which was adjusted by purity. Similar to TIMER data analysis, we also found that there was significant positive correlation of ZC3H13 with PD1, PD-L1, or CTLA-4 in HCC. Additionally, a strong correlation exists between PD-L1 and ZC3H13. These results demonstrate that tumor immune escape might be involved in $\mathrm{ZC} 3 \mathrm{H} 13$ mediated carcinogenesis of HCC.

\section{Discussion}

To date, HCC is still notorious for its poor prognosis. Elucidating the molecular mechanism of HCC carcinogenesis may provide key clues for developing effective therapeutic targets or seeking promising prognostic biomarkers. Increasing evidence has demonstrated that ZC3H13 plays key roles in initiation and progression of multiple human cancers, including HCC. In previous study, ZC3H13 may be an upstream regulator of Ras-ERK signaling pathway and suppressed invasion and proliferation of colorectal cancer [12]. And Huang et al study first identified an RBP-related six-gene prognostic signature, which could serve as a promising prognostic biomarker and provide some potential therapeutic targets for HCC [20]. Joint effects analysis showed the predictive capacity of combining METTL3, YTHDF2, and ZC3H13 for HCC OS [21]. Several studies reported ZC3H13 loss expression in HCC, but the mechanisms that lead to $\mathrm{ZC} 3 \mathrm{H} 13$ silencing are still unknown.

In this study, we first conducted pan-cancer analysis of ZC3H13's expression using Oncomine and TCGA databases to jointly analyze the expression of ZC3H13. Survival analysis for ZC3H13 in those cancer types of interest indicated that HCC patients with low expression of $\mathrm{ZC} 3 \mathrm{H} 13$ had poor prognosis. These results reflect those of $\mathrm{Wu}, \mathrm{X}$. et al. [22], who also found that $\mathrm{ZC} 3 \mathrm{H} 13$ showed low expression in LIHC. This report together with our analytic results showed the significant interest of ZC3H13 in HCC. It has been well documented that ncRNAs, including miRNAs, IncRNAs, and circular RNAs (circRNAs), participated in regulation of gene expression by talking with each other through the ceRNA mechanism [23]. To explore the upstream regulatory miRNAs of $\mathrm{ZC} 3 \mathrm{H} 13$, we introduced seven prediction programs, involving PITA, RNA22, miRmap, microT, miRanda, PicTar, and TargetScan, to predict possible miRNAs that could potentially bind to $\mathrm{ZC} 3 \mathrm{H} 13$. At the end, 12 miRNAs were finally obtained. Most of these miRNAs have been found to act as oncogene miRNAs in HCC. For example, miR-425-5p as an oncogene promotes the malignant development of HCC via RNF11 and serves as a molecular target for predicting the prognosis of HCC patients [24]. Upregulation of miR-362-3p modulates proliferation and anchorageindependent growth by Directly Targeting Tob2 in hepatocellular carcinoma [25]. After performing 
correlation analysis, expression analysis, and survival analysis, miR-362-3p/miR-425-5p were selected as the most potential upstream oncogene miRNA of $\mathrm{ZC} 3 \mathrm{H} 13$. Previous studies also showed that miR-362$3 p / m i R-425-5 p$ played oncogene roles in modulating proliferation and migration of HCC. For example, Li, $Z$ et al. found that knockdown of circMYLK and overexpressed miR-362-3p could suppress the expression of Rab23, thus inhibiting the growth and proliferation of Hep3B cells in vivo [26]. miR-425-5p as an oncogene promotes the malignant development of HCC via RNF11 and serves as a molecular target for predicting the prognosis of HCC patients.

In the following respect, the mechanism of downregulation of $\mathrm{ZC} 3 \mathrm{H} 13$ in $\mathrm{LIHC}$ was mainly explained. We discovered that miR-362-3p/miR-425-5p binds to the ZC3H13 3' UTR(Additional file 3), validating databases findings that miR-362-3p/miR-425-5p targets and regulates ZC3H13 expression. Additionally, miR-362-3p/miR-425-5p expression was inversely associated with ZC3H13 expression in LIHC tissues. $\mathrm{Zhu}, \mathrm{D}$ et al. found that $\mathrm{ZC} 3 \mathrm{H} 13$ was served as a tumor suppressor in colorectal cancer cells, which decreased the expression of Snail, Cyclin D1, and Cyclin E1, and increased the expression of Occludin and Zo-1 through inactivating Ras-ERK signaling pathway [27].

Numerous studies have confirmed that tumor immune cell infiltration could influence the efficacies of chemotherapy, radiotherapy, or immunotherapy and prognosis of cancer patients. Our work suggested that $\mathrm{ZC} 2 \mathrm{H} 13$ was significantly positively correlated with various immune cells, including CD4+T cell, macrophage, neutrophil, and dendritic cell in HCC. Moreover, ZC3H13 was also markedly positively associated with biomarkers of these infiltrated immune cells. These findings indicated that tumor immune infiltration might partially account for $\mathrm{ZC} 3 \mathrm{H} 13$-mediated tumor suppressor gene roles in HCC.

In summary, we elucidated that $\mathrm{ZC} 3 \mathrm{H} 13$ was obviously decreased and positively correlated with unfavorable prognosis in HCC. We identified an upstream regulatory mechanism of ZC3H13 in HCC, namely miR-362-3p/miR-425-5p-ZC3H13 axis. Furthermore, our current findings also indicated that ZC3H13 might exert tumor suppressor gene through increasing tumor immune cell infiltration and positively associated with biomarkers of these infiltrated immune cells. However, these results should be validated by much more basic experiments and large clinical trials in the future.

\section{Declarations}

\section{Funding}

This work was supported by National Natural Science Foundation of China (Grant No. 81402579), Natural Science Foundation of Shandong Province General Project (Grant No. ZR2020MH318), the Key Program of Research and Development Foundation of Shandong province (Grant No. 2017GSF18179), Source Innovation Foundation of Qingdao (Grant No. 18-2-2-79-jch) and "Clinical Medicine $+X$ " of Qingdao University (Grant No. CMX201729).

\section{Acknowledgments}


The authors thank the contributors of The Cancer Genome Atlas for their contribution to share the sequencing dataset on open access. The authors thank the contributors of related databases for their contribution to facilitate the analyses of The Cancer Genome Atlas datasets.

\section{Data Availability}

The datasets presented in this study can be found in online repositories. The names of the repository can be found in the article.

\section{Declarations}

\section{Ethics approval and consent to participate}

Not applicable.

\section{Consent for publication}

Not applicable.

\section{Competing Interests}

The authors declare that there are no competing interests associated with the manuscript.

\section{Author contributions}

SW, and SHL conceived the project and wrote the manuscript. SW, SHL and YXC participated in data analysis. GC, and PW participated in discussion and language editing. HZP reviewed the manuscript. All authors contributed to the article and approved the submitted version.

\section{References}

1. Erratum: Global cancer statistics 2018: GLOBOCAN estimates of incidence and mortality worldwide for 36 cancers in 185 countries. CA: a cancer journal for clinicians 2020, 70(4):313.

2. Llovet J, Kelley R, Villanueva A, Singal A, Pikarsky E, Roayaie S, Lencioni R, Koike K, Zucman-Rossi J, Finn R: Hepatocellular carcinoma. Nature reviews Disease primers 2021, 7(1):6.

3. Chen HY, Chen YM, Wu J, Yang FC, Lv Z, Qian YG, Zheng SS: Effects of HGF gene polymorphisms and protein expression on transhepatic arterial chemotherapeutic embolism efficacy and prognosis in patients with primary liver cancer. Onco Targets Ther 2017, 10:803-810. 
4. Zhu XD, Sun HC: Emerging agents and regimens for hepatocellular carcinoma. J Hematol Oncol 2019, 12(1):110.

5. de Jesus VHF, Dettino ALA: Update on hepatocellular carcinoma from the $\mathbf{2 0 1 8}$ Gastrointestinal Cancer Symposium (ASCO GI). J Hepatocell Carcinoma 2018, 5:87-90.

6. Geula S, Moshitch-Moshkovitz S, Dominissini D, Mansour A, Kol N, Salmon-Divon M, Hershkovitz V, Peer E, Mor N, Manor $Y$ et al: Stem cells. m6A mRNA methylation facilitates resolution of naïve pluripotency toward differentiation. Science (New York, NY) 2015, 347(6225):1002-1006.

7. He L, Li H, Wu A, Peng Y, Shu G, Yin G: Functions of N6-methyladenosine and its role in cancer. Mol Cancer 2019, 18(1):176.

8. Du K, Zhang L, Lee T, Sun T: mA RNA Methylation Controls Neural Development and Is Involved in Human Diseases. Molecular neurobiology 2019, 56(3):1596-1606.

9. Meyer K, Jaffrey SJAroc, biology d: Rethinking mA Readers, Writers, and Erasers. 2017, 33:319-342.

10. Knuckles P, Lence T, Haussmann IU, Jacob D, Kreim N, Carl SH, Masiello I, Hares T, Villaseñor R, Hess $\mathrm{D}$ et al: Zc3h13/Flacc is required for adenosine methylation by bridging the mRNA-binding factor $\mathrm{Rbm15/Spenito} \mathrm{to} \mathrm{the} \mathrm{m(6)A} \mathrm{machinery} \mathrm{component} \mathrm{Wtap/FI(2)d.} \mathrm{Genes} \mathrm{\&} \mathrm{development} \mathrm{2018,} \mathrm{32(5-}$ 6):415-429.

11. Gong PJ, Shao YC, Yang Y, Song WJ, He X, Zeng YF, Huang SR, Wei L, Zhang JW: Analysis of N6Methyladenosine Methyltransferase Reveals METTL14 and ZC3H13 as Tumor Suppressor Genes in Breast Cancer. Front Oncol 2020, 10:578963.

12. Zhu D, Zhou J, Zhao J, Jiang G, Zhang X, Zhang Y, Dong M: ZC3H13 suppresses colorectal cancer proliferation and invasion via inactivating Ras-ERK signaling. Journal of cellular physiology 2019, 234(6):8899-8907.

13. Rhodes D, Kalyana-Sundaram S, Mahavisno V, Varambally R, Yu J, Briggs B, Barrette T, Anstet M, Kincead-Beal C, Kulkarni $P$ et al: Oncomine 3.0: genes, pathways, and networks in a collection of 18,000 cancer gene expression profiles. 2007, 9(2):166-180.

14. Tang Z, Li C, Kang B, Gao G, Li C, Zhang Z: GEPIA: a web server for cancer and normal gene expression profiling and interactive analyses. Nucleic acids research 2017, 45:W98-W102.

15. Hou G, Liu P, Yang J, Wen S: Mining expression and prognosis of topoisomerase isoforms in nonsmall-cell lung cancer by using Oncomine and Kaplan-Meier plotter. PloS one 2017, 12(3):e0174515.

16. Yang JH, Li JH, Shao P, Zhou H, Chen YQ, Qu LH: starBase: a database for exploring microRNAmRNA interaction maps from Argonaute CLIP-Seq and Degradome-Seq data. Nucleic Acids Res 2011, 39(Database issue):D202-209.

17. Li T, Fan J, Wang B, Traugh N, Chen Q, Liu JS, Li B, Liu XS: TIMER: A Web Server for Comprehensive Analysis of Tumor-Infiltrating Immune Cells. Cancer Res 2017, 77(21):e108-e110.

18. Vasaikar SV, Straub P, Wang J, Zhang B: LinkedOmics: analyzing multi-omics data within and across 32 cancer types. Nucleic Acids Res 2018, 46(D1):D956-d963. 
19. Chandrashekar DS, Bashel B, Balasubramanya SAH, Creighton CJ, Ponce-Rodriguez I, Chakravarthi B, Varambally S: UALCAN: A Portal for Facilitating Tumor Subgroup Gene Expression and Survival Analyses. Neoplasia 2017, 19(8):649-658.

20. Huang Y, Chen S, Qin W, Wang Y, Li L, Li Q, Yuan X: A Novel RNA Binding Protein-Related Prognostic Signature for Hepatocellular Carcinoma. Frontiers in oncology 2020, 10:580513.

21. Liu GM, Zeng HD, Zhang CY, Xu JW: Identification of METTL3 as an Adverse Prognostic Biomarker in Hepatocellular Carcinoma. Digestive diseases and sciences 2021, 66(4):1110-1126.

22. Wu X, Zhang X, Tao L, Dai X, Chen P: Prognostic Value of an m6A RNA Methylation Regulator-Based Signature in Patients with Hepatocellular Carcinoma. Biomed Res Int 2020, 2020:2053902.

23. Fiannaca A, Paglia L, Rosa M, Rizzo R, Urso A: miRTissue: extending miRTissue web service with the analysis of ceRNA-ceRNA interactions. BMC bioinformatics 2020, 21:199.

24. Rao D, Guan S, Huang J, Chang Q, Duan S: miR-425-5p Acts as a Molecular Marker and Promoted Proliferation, Migration by Targeting RNF11 in Hepatocellular Carcinoma. Biomed Res Int 2020, 2020:6530973.

25. Shen H, Li W, Tian Y, Xu P, Wang H, Zhang J, Li Y: Upregulation of miR-362-3p Modulates Proliferation and Anchorage-Independent Growth by Directly Targeting Tob2 in Hepatocellular Carcinoma. Journal of cellular biochemistry 2015, 116(8):1563-1573.

26. Li Z, Hu Y, Zeng Q, Wang H, Yan J, Li H, Yu Z: Circular RNA MYLK promotes hepatocellular carcinoma progression by increasing Rab23 expression by sponging miR-362-3p. Cancer cell international 2019, 19:211.

27. Zhu D, Zhou J, Zhao J, Jiang G, Zhang X, Zhang Y, Dong M: ZC3H13 suppresses colorectal cancer proliferation and invasion via inactivating Ras-ERK signaling. Journal of cellular physiology 2019, 234(6):8899-8907.

\section{Figures}


A

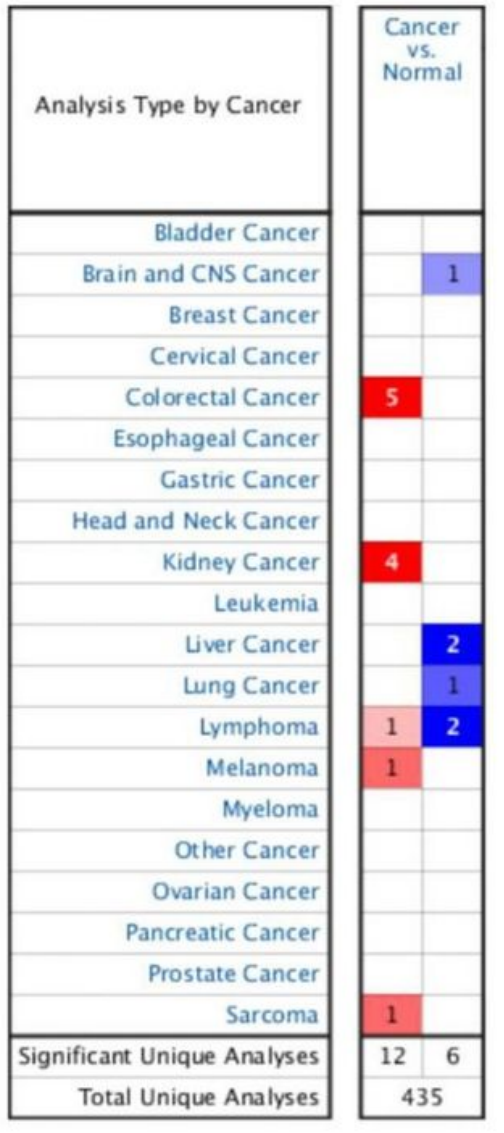

\section{불ำ}

B

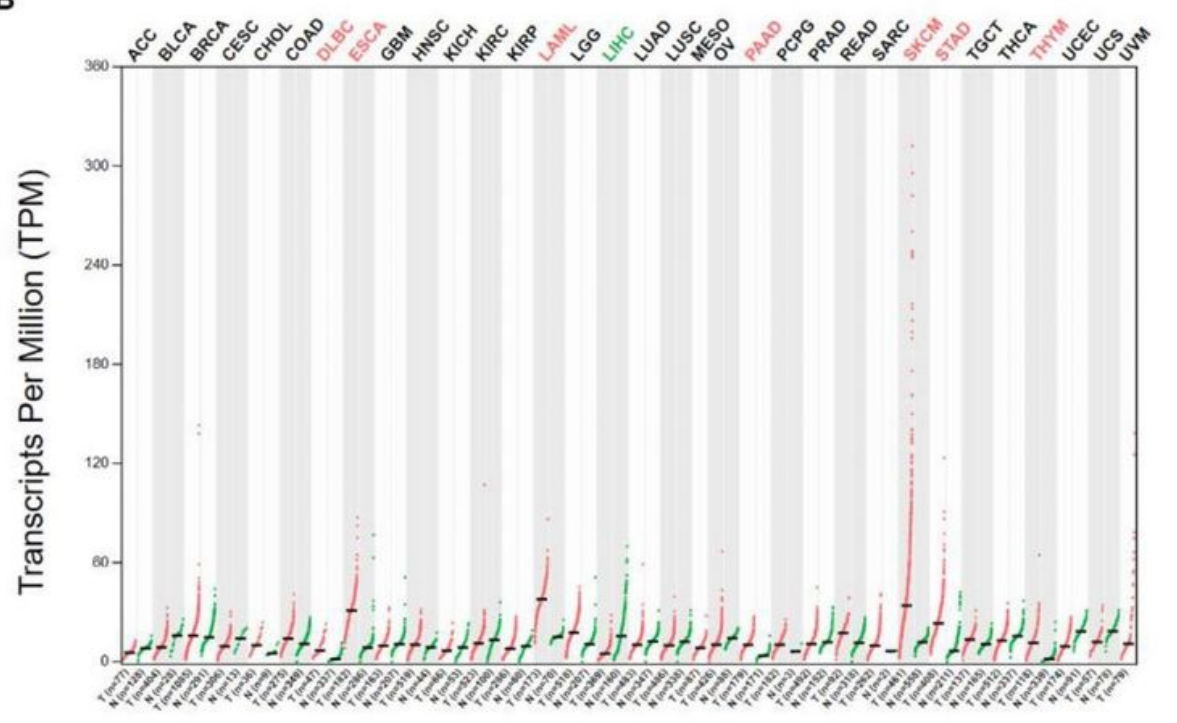

C

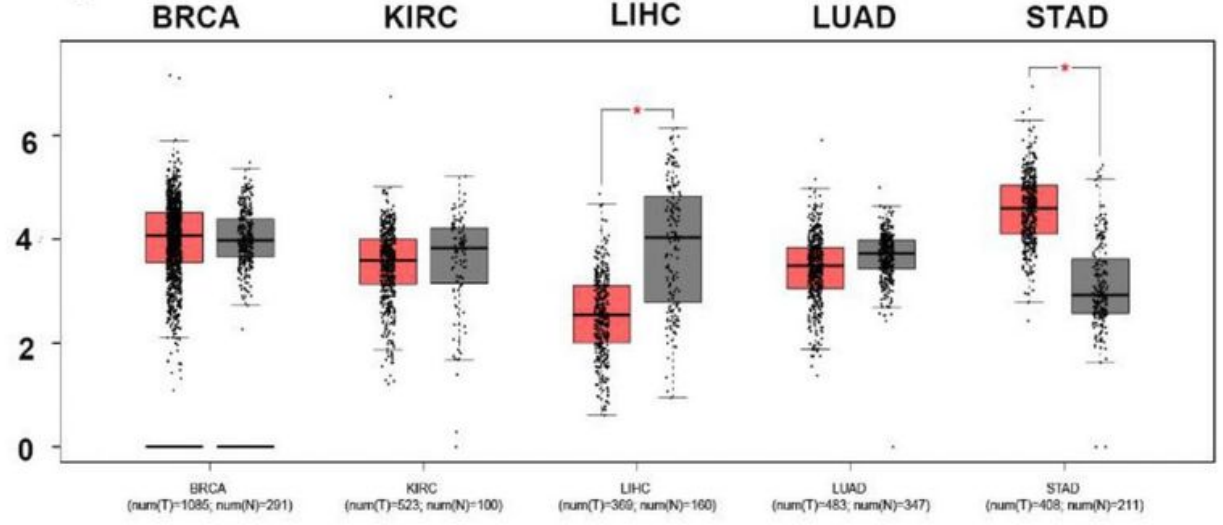

\section{Figure 1}

Expression analysis for ZC3H13 in multiple cancers. (A) mRNA expression levels of ZC3H13 in various types of cancer (Oncomine). The threshold was designed with following parameters: fold change $=2$ and P-value $=0.01$. The cell number represents the number of datasets that meets the thresholds. The color intensity (red or blue) is directly proportional to the significance level of upregulation or downregulation, respectively. (B-C) ZC3H13 expression in TCGA (BRCA, KIRC, LIHC, LUAD, STAD) tissues compared with corresponding TCGA and GTEx normal tissues. ${ }^{*} p$ value $<0.05$. 

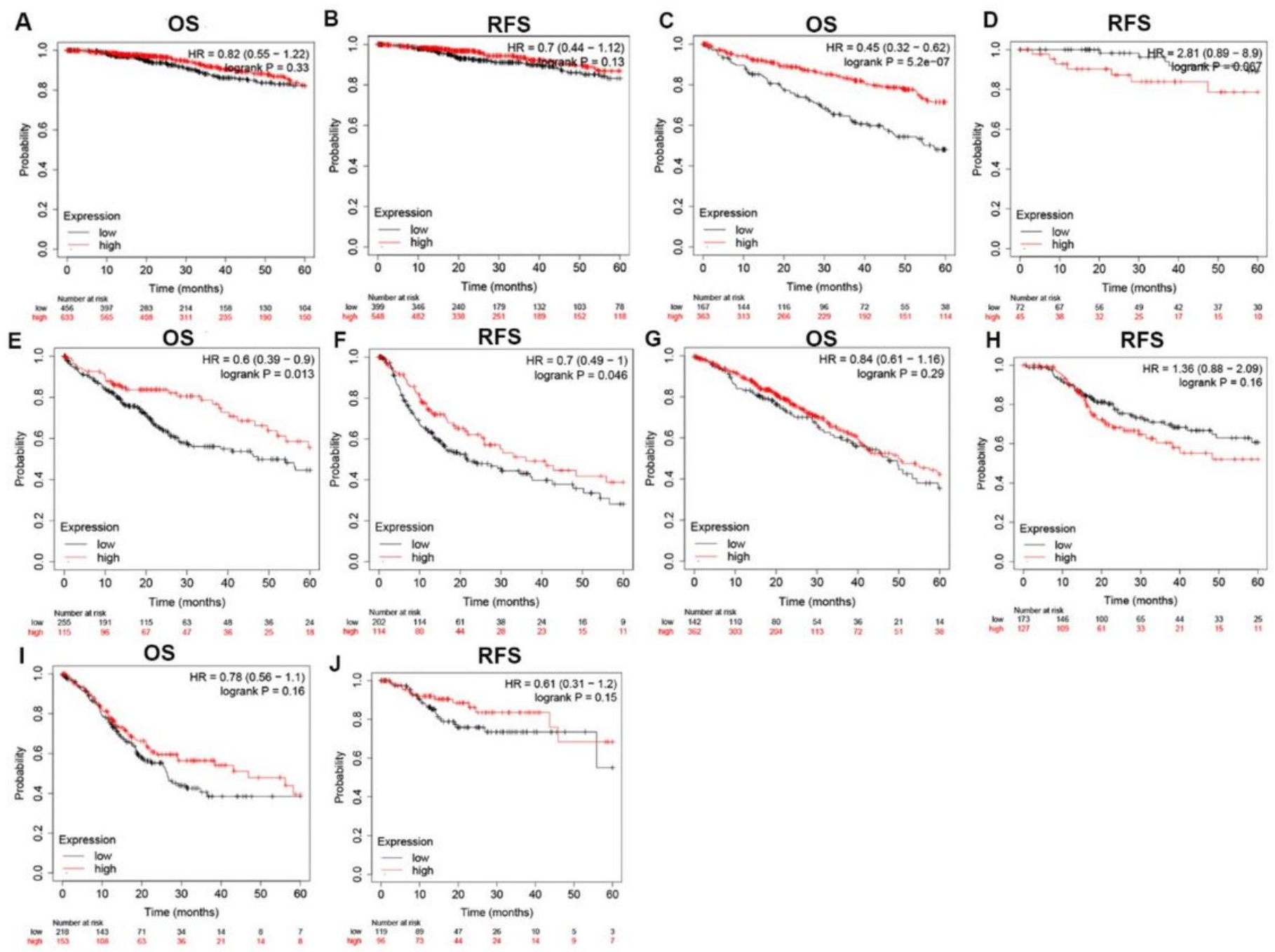

\section{Figure 2}

The overall survival (OS) and disease-free survival (RFS) analysis for ZC3H13 in various human cancers determined by GEPIA database. The OS plot of ZC3H13 in BRCA (A), KIRC (C), LIHC (E), LUAD (G), and STAD (I). The RFS plot of ZC3H13 in BRCA (B), KIRC (D), LIHC (F), LUAD (H), and STAD (J). 
A

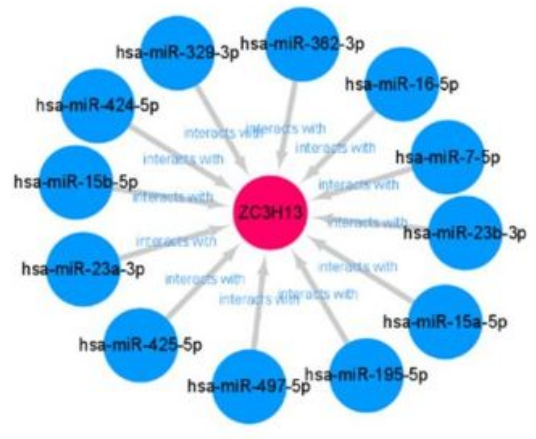

E

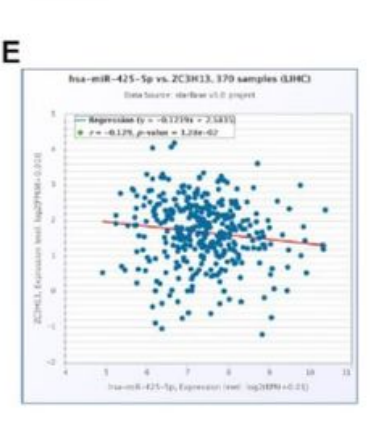

C

B



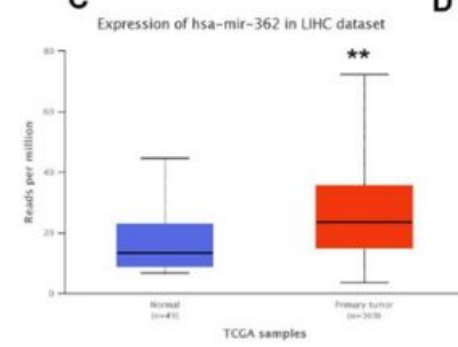
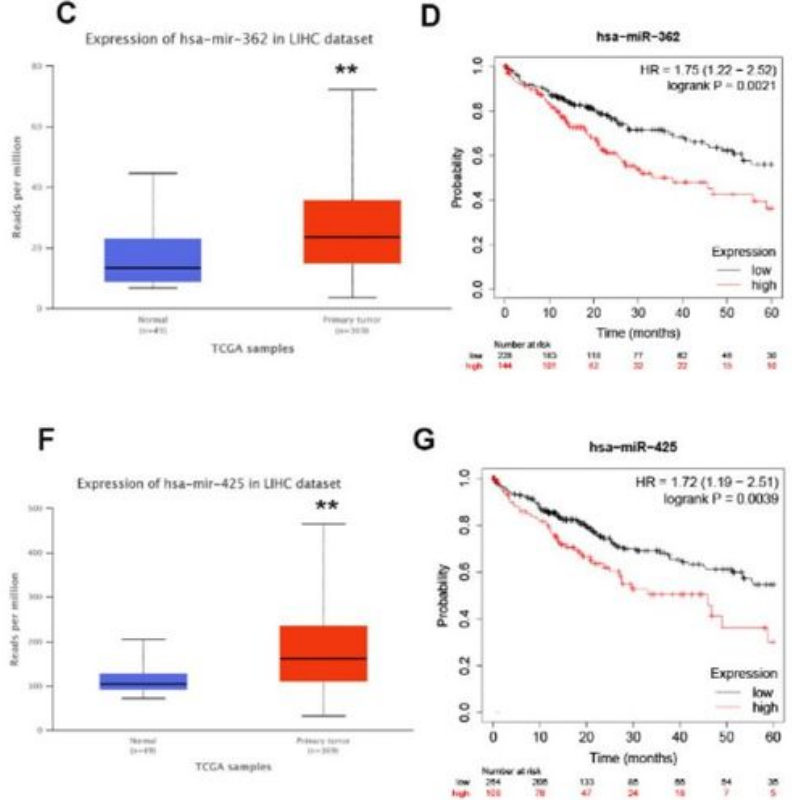

H





J
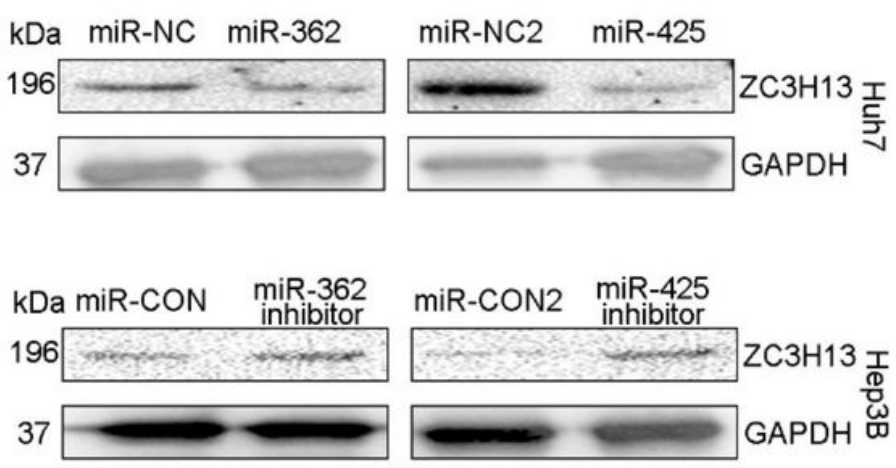

\section{Figure 3}

MiR-362-3p/miR-425-5p downregulates ZC3H13 in liver cancer. (A) The miRNA-ZC3H13 regulatory network established by cytoscape software. (B) ZC3H13 is negatively correlated with miR-362-3p in LIHC analyzed by StarBase database. (C) MiR-362-3p is overexpressed in LIHC analyzed by UALCAN. (D) The prognostic value of miR-362-3p in HCC assessed by Kaplan-Meier plotter. (E) ZC3H13 is negatively correlated with miR-425-5p in LIHC analyzed by StarBase database. (F) MiR-425-5p is overexpressed in LIHC analyzed by UALCAN. (G) The prognostic value of miR-425-5p in HCC assessed by Kaplan-Meier plotter. $(\mathrm{H}, \mathrm{I})$ MiR-362-3p/miR-425-5p mimics and inhibitors results detected by qPCR. (J) MiR-362$3 p / m i R-425-5 p$ and inhibitors results detected by western blotting. ${ }^{*} P<0.01$. 
A

ZC3H13 Association Result



B

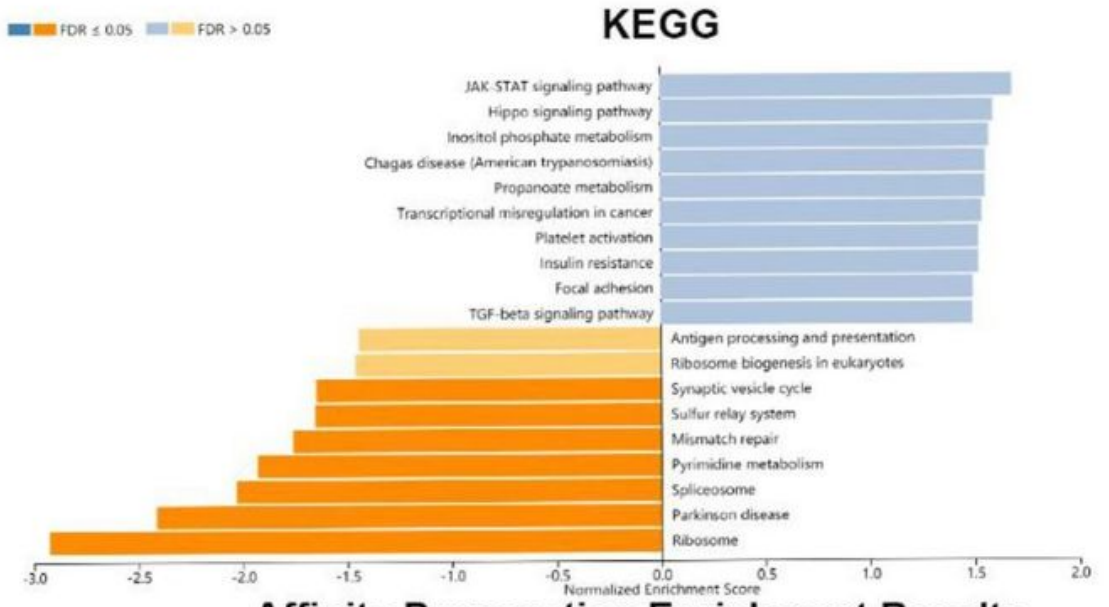

Affinity Propagation Enrichment Results

C
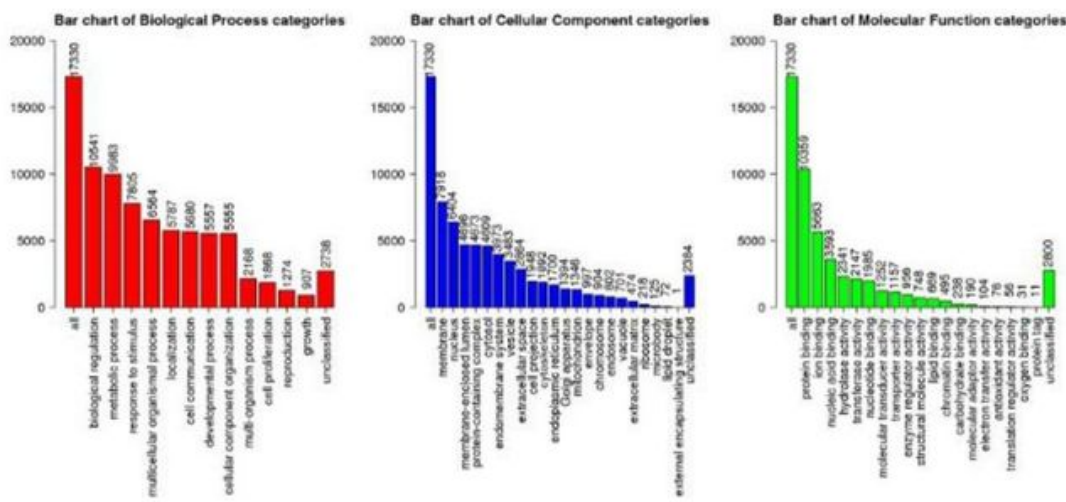

\section{Figure 4}

ZC3H13 co-expression genes in LIHC (LinkedOmics). (A) The global ZC3H13 highly correlated genes identified by the Pearson test in LIHC. Red and green dots represent positively and negatively significantly correlated genes with ZC3H13, respectively. (B) KEGG pathways of ZC3H13 correlated genes, respectively. (C) Gene Ontology of ZC3H13 correlated genes, respectively. KEGG: Kyoto Encyclopedia of Genes and Genomes; LIHC: Liver hepatocellular carcinoma. 

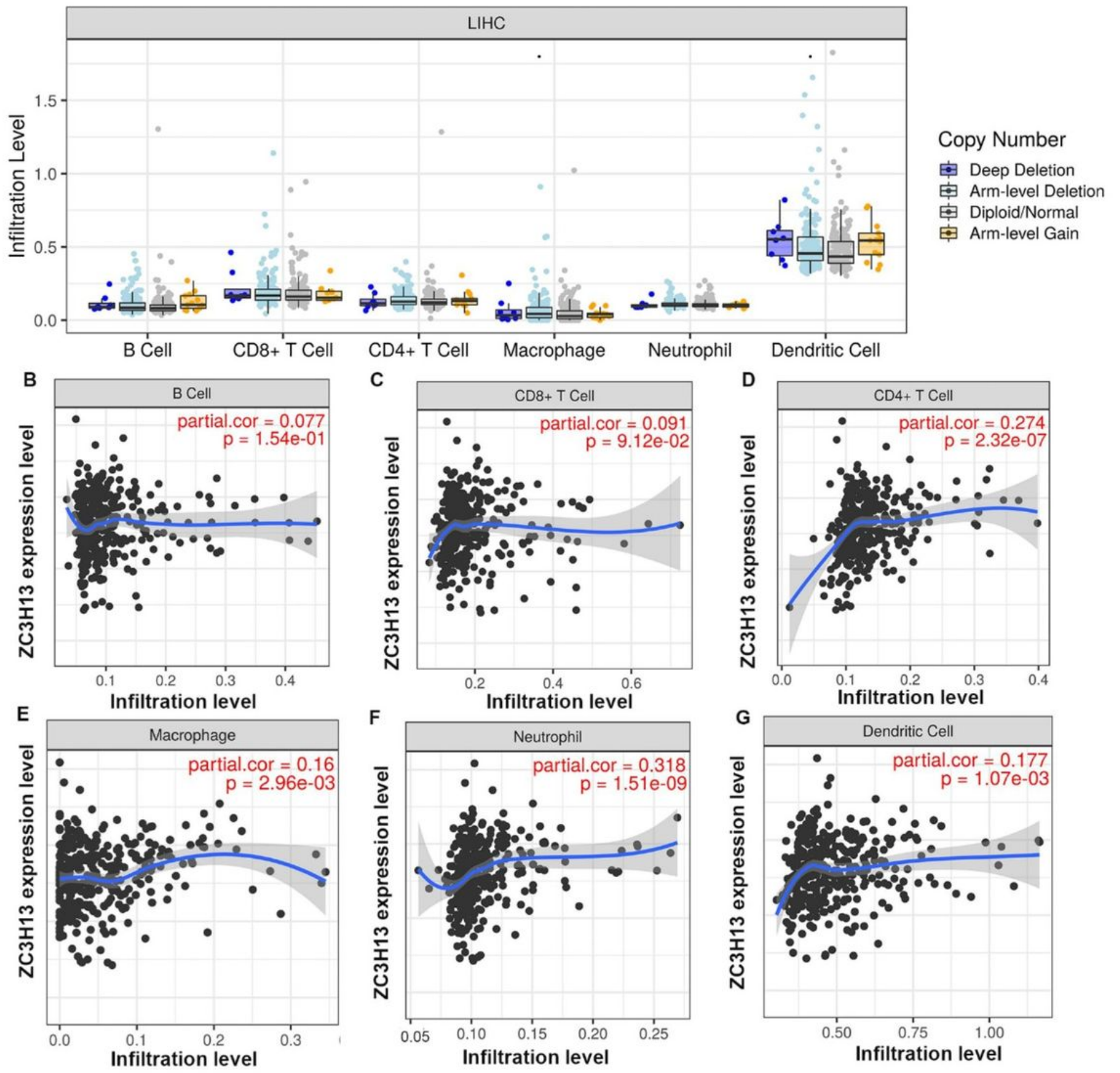

\section{Figure 5}

(A)The relationship of immune cell infiltration with $\mathrm{ZC} 3 \mathrm{H} 13$ level in $\mathrm{HCC}$. $(\mathrm{B}-\mathrm{G})$ The infiltration level of various immune cells under different copy numbers of ZC3H13 in HCC. The correlation of ZC3H13 expression level with $B$ cell $(B), C D 8+T$ cell $(C), C D 4+T$ cell $(D)$, macrophage (E), neutrophil (F), or dendritic cell $(G)$ infiltration level in HCC.

\section{Supplementary Files}


This is a list of supplementary files associated with this preprint. Click to download.

- GraphicalAbstarct.jpg

- Additionalfiles.docx 